\title{
Developing nurse match: An instrument for assessing personal nursing values against a set of preferred nursing values
}

\author{
Elaine Hogard ${ }^{1}$ and Roger Ellis ${ }^{2 *}$ \\ ${ }^{1}$ Northern Ontario School of Medicine, Canada \\ ${ }^{2}$ University of Chester, University of Ulster, and Buckinghamshire New University, UK
}

\begin{abstract}
This paper reports the development of Nurse Match (NM) a values-based self-report instrument for assessing the nature, relative importance and emotional significance of personal values about nursing held by nurses, nursing students or those aspiring to be nurses. The NM instrument is intended initially for use in Value Based Recruitment (VBR but can also be an aid for the charting of professional development in initial training and professional development. VBR has been introduced in the UK to address the concern that standards in nursing may be falling with students and practitioners not having the right nursing values. The development of the NM instrument and its theoretical and methodological background was first reported by Ellis et al. [1] and reference is made to that below. The instrument requires respondents to apply constructs to entities and these were chosen to indicate Nursing values. These values were derived from the literature; expert views; and most recently the preferred attributes based on NIPEC research [2], and then combined by NIPEC into six nursing values. The primary aim of the work described in this paper was to continue development of NM by piloting the instrument with a cohort of nursing students, scoring the resulting value profiles, refining scoring protocols; and obtaining feedback on user experience. A secondary aim was to explore the relationship between cohort scores on the pilot instrument and on a number of other measures of nursing competence including personal statements, selection interviews and a Multiple Mini Interview (MMI) selection process. Administration of the instrument and its scoring worked well and the scoring process for comparing respondent profiles on nursing values was refined. The instrument discriminated effectively between the nursing students responding and results conform to a normal distribution. The secondary aim of correlating with existing measures was inconclusive and is a work in progress with initial indications being of low or no correlations between the various measures of nursing values and competence. The instrument has face and content validity identifying the important nursing values, was interesting to respondents and easy to understand and complete. As a self-report measure it was considered to be superior to the personal statement used widely at present in VBR Nursing recruitment.
\end{abstract}

\section{Introduction}

Values Based Recruitment (VBR) is an important programme of work within the UK National Health Service. It was devised after a mandate DH [3] from government to Health Education England (HEE) to deliver high quality, effective, compassionate care: developing the right people with the right skills and the right values. The emphasis on the 'right' values is a response to a widespread concern that those recruited to nursing might not develop and evidence appropriate professional behaviour and values.

VBR is an approach which recruits students, trainees or employees on the basis that their individual values and behaviours align with the values of the NHS Constitution [4]. It is about enhancing existing processes to ensure that the NHS recruits the right workforce not only with the right skills and in the right numbers, but with the right values to support effective team working and excellent patient care and experience HEE [5].

There has been an increasing focus on the values agenda across the NHS, in part due to the Francis report [6] which highlighted the vital role of the workforce in providing high quality and safe healthcare. In particular, the report emphasised the importance of staff values and behaviours for the level of care and patient experience $[7,8]$.

The Department of Health, Social Services and Public Safety
(DHSSPS) Education Strategy Group (ESG) identified a need for streamlining the application and selection processes for Higher Education Institutions (HEIs) during 2011. The Northern Ireland Practice and Education Council for Nursing and Midwifery (NIPEC) was commissioned by the ESG to undertake a project to develop a strategy which would optimise efficiency of application and selection processes to identify individuals who display attributes that are valued.

Phase Two of that project focussed on the 'attributes which are valued to realise future potential in a career in nursing'. The NIPEC report to ESG, NIPEC [2], considered that it had 'added to the growing evidence in relation to the attributes that could be used in selecting students'. The values and attributes used in the NIPEC project are set out in NIPEC [2], at Appendix A and B.

Ellis et al. [1] reported the development of a new instrumentNurse Match-to measure the nursing values held by respondents. The instrument was intended to support the value based recruitment of student nurses. Whilst other instruments existed purporting to

Correspondence to: Roger Ellis,, Social and Health Evaluation Unit, School of Nursing, Ulster University, UK, E-mail: rogerellis2@icloud.com

Received: May 01, 2016; Accepted: May 16, 2016; Published: May 19, 2016 
measure relevant values, none had been dedicated specifically to nursing values. Other methods used to gauge values including the assessment of a personal statement were considered to be of doubtful validity and reliability.

Ellis et al. [1] located the new values based instrument, theoretically and empirically in a recent review of available instruments to measure identity and hence values [9]. From the wide range of instrument and approaches they considered Passmore et al recommended Weinreich's Identity Structure Analysis (ISA) and instruments constructed in its framework using the associated Ipseus software. It was therefore decided to construct the Nurse Match instrument using this approach the utility of which had been demonstrated in a number of studies described in Weinreich and Saunderson [10].

An Ipseus instrument requires respondents to apply a number of bipolar constructs to entities representative of the self and its social world. These constructs and entities are chosen to reflect the salient features of the area being studied. In this case the bipolar constructs were chosen to represent key nursing values. The paper listed the nursing values used to determine the constructs in the new instrument, called Nurse Match (NM), and their derivation from the literature and interviews and focus groups with expert members of the nursing profession. The paper demonstrates how the instrument offers an in depth analysis of the respondent's position regarding key nursing values and how initial results using an early version of the instrument demonstrated its power to identify and distinguish value orientations of individuals.

The primary objective of the follow-up work described in this paper was therefore the development and assessment of the Nurse Match instrument to ensure that it was a feasible and relatively simple measure easily understood and managed. The results recorded through the instruments software had to provide a way of systematically appraising a respondent's personal nursing values against a set of values preferred by the profession. In this sense the instrument allowed respondents responses to be 'matched' with an ideal set as agreed by the profession. In this case the profession's view was captured in the six NIPEC values or 'themed attributes' (Table 1).

It was also important to confirm that the instrument appeared valid to respondents as well as relatively easy to understand and complete and so feedback was sought from the respondents.

A secondary objective was to explore the relationship between cohort scores on the pilot instrument and on a number of other measures of nursing values; personal statements, selection interviews and an MMI selection process.

\section{Method}

\section{Measures}

Two measures were used.

1. The Nurse Match (NM) instrument consisting of 20 bi-polar nursing values and 13 entities constructed and presented on Ipseus software (Table 2 and 3 ).

\section{A Feedback Questionnaire (Appendix C).}

The values used in the NM instrument were derived from a literature search, interviews with expert nurses individually and in groups; ad trials with experienced and well respected nurses. They were aligned with NIPEC attributes and values. Each was presented
Table 1. The entities used in Nurse Mismatch.

\begin{tabular}{|l|l|}
\hline & Entity wording \\
\hline 01 & Ideal self \\
\hline 02 & Real work self \\
\hline 03 & Real home self \\
\hline 04 & Real self, under pressure \\
\hline 05 & Real self, two years ago \\
\hline 06 & Real self, in five years' time \\
\hline 07 & The person I most dislike \\
\hline 08 & A model nurse \\
\hline 09 & A ward sister \\
\hline 10 & A typical patient \\
\hline 11 & A bad nurse \\
\hline 12 & My best friend \\
\hline 13 & My parents \\
\hline
\end{tabular}

as a bi-polar construct offering alternative attitudes to the value. One pole of each construct had been identified as the preferred option from a professional nursing point of view. The entities are aspects of self and people from the workplace and home context.

The software presented each respondent with a nine-point, semantic differential scale with centre zero. A click on the preferred pole scored from 1 to 4 and on the alternative pole from -1 to -4 . The centre zero was used by the respondent if they could not decide between polar values.

Respondents were asked to apply the constructs to all aspects of self (including aspirational self) and all other people in terms of the attitudes or values they perceive them to hold (e.g. at work I ... am prepared to challenge someone more senior if I feel it is in the interests of the patient/would not challenge someone more senior in any circumstances).

Data output was presented as an Ipseus report which was produced by the software based on the judgements made by the respondent. The Ipseus report covers a number of identity parameters concerning use of constructs derived from the theoretical framework of Identity Structure Analysis (ISA). For the NM instrument the parameters focussed upon were: choice of pole for each construct; structural pressure (stability) that is their importance and significance in making judgements; and the emotional loading of each construct. These were used to calculate a score (S) for each of the twenty NM nursing values (see Appendix B for the details of the calculation).

Each of the six value themes (Person Centeredness (PC), Accountability (ACC), Trust (T), Integrity (I), Commitment to Personal Development (CPD), Teamwork (TW), is composed of a set of NM values or attributes (Table 3). The attributes and themes they were alligned with were recommended by the partner School of Nursing having proved their worth in Phase Two of the ERC projectNIPEC [2] at Appendix C.

The score for each major value $\left(\mathrm{S}^{\mathrm{TOT}}\right)$ is the sum of the $\mathrm{S}$ scores on the constituent NM values. The mean of the six $S^{\text {TOT }}$ scores is the individual's score on NM for assessment purpose see Appendix A: Table 3 for the calculation.

The second measure used was a feedback questionnaire (Appendix C). It was completed by all respondents immediately following completion of the instrument. A free text box was available.

\section{Respondents}

The respondents $(\mathrm{N}=63)$ were first year students at the School of 
Table 2. The bi-polar dimensions of value used in NM.

\begin{tabular}{|c|c|c|}
\hline & Preferred option & Alternative option \\
\hline 01 & can critically assess their own thinking and behaviour & finds it difficult to critically assess their own thinking and behaviour \\
\hline 02 & believes that patient dignity and human rights must take precedence at all times & $\begin{array}{l}\text { feels that there are times when the patient's dignity and human rights should be } \\
\text { temporarily set aside }\end{array}$ \\
\hline 03 & listens carefully and is tuned into the needs of patients and work colleagues & finds listening a distraction and prefers just to get on with the job \\
\hline 04 & is an excellent communicator and is always understood & is a poor communicator and is often misunderstood \\
\hline 05 & usually understands and relates well to others & often seems to misunderstand and have problems relating to people \\
\hline 06 & can be relied upon to deliver on their commitments & is only human and may not meet commitments due to real world constraints \\
\hline 07 & manages time and workloads with little supervision & works best when being managed by someone \\
\hline 08 & is open and honest at all times & will sometimes keep information from others in the greater interest of all concerned \\
\hline 09 & generally understands people and situations & sometimes misunderstands people and situations \\
\hline 10 & $\begin{array}{l}\text { is prepared to challenge someone more senior if they feel it is in the interests of the } \\
\text { patient }\end{array}$ & would not challenge someone more senior in any circumstances \\
\hline 11 & owns their work and takes personal responsibility for their decisions and actions & adheres strictly to guidelines and instructions, which are at fault if things go wrong \\
\hline 12 & enjoys making decisions within their area of competence & in a shared area of competence prefers the other person to take decisions \\
\hline 13 & has no difficulty influencing people and getting them to follow instructions & finds it hard to influence people and get them to follow instructions \\
\hline 14 & would take all the time needed to do a task properly & would sometimes take shortcuts in the interests of saving time \\
\hline 15 & often pauses and reflects on how things have gone & rarely takes time to reflect on how things have gone \\
\hline 16 & works hard to continue their learning and development throughout their career & $\begin{array}{l}\text { thinks that learning is for student nurses and qualified nurses should focus on } \\
\text { delivering nursing care }\end{array}$ \\
\hline 17 & is always thinking about the other person & focuses on own needs and priorities \\
\hline 18 & prefers to achieve things by working closely with others in a medical team & prefers to achieve things through individual initiative \\
\hline 19 & believes that the safety of patients must come before everything else & $\begin{array}{l}\text { accepts that realistically patient safety will sometimes suffer as a result of pressures on } \\
\text { the health service }\end{array}$ \\
\hline 20 & $\begin{array}{l}\text { believes that resource constraints are no excuse for a lack of kindness, compassion and } \\
\text { sympathy }\end{array}$ & $\begin{array}{l}\text { accepts that resource constraints mean that modern nursing must prioritise technical } \\
\text { and medical competencies over kindness compassion and sympathy }\end{array}$ \\
\hline
\end{tabular}

Table 3. Scoring matrix for six value themes assessed using NM construct values.

\begin{tabular}{|c|c|c|c|c|c|c|c|}
\hline Constructs (20) & $\begin{array}{c}\text { Person } \\
\text { Centeredness (PC) }\end{array}$ & $\begin{array}{c}\text { Account } \\
\text { Ability (ACC) }\end{array}$ & Trust (T) & Integrity (I) & $\begin{array}{c}\text { Commitment } \\
\text { Personal development (CPD) }\end{array}$ & $\begin{array}{c}\text { Team } \\
\text { Work (TW) }\end{array}$ & $\begin{array}{r}\text { Tota } \\
\text { Use }\end{array}$ \\
\hline 1 & $X-1$ & $X-1$ & $X-1$ & & & & 3 \\
\hline 2 & $X-1$ & $X-1$ & $X-1$ & & & & 3 \\
\hline 3 & & $X-1$ & & & & $X-1$ & 2 \\
\hline 4 & & & & & & $X-1$ & 1 \\
\hline 5 & $X-1$ & $X-1$ & & & & & 2 \\
\hline 6 & & & & & $X-1$ & & 1 \\
\hline 7 & $X-1$ & & & & & & 1 \\
\hline 8 & & $X-1$ & & $X-1$ & & & 2 \\
\hline 9 & $X-1$ & $X-1$ & & & & & 2 \\
\hline 10 & & $X-1$ & & $X-1$ & & & 2 \\
\hline 11 & & $X-1$ & & & $X-1$ & & 2 \\
\hline 12 & & $X-1$ & $X-1$ & & $X-1$ & & 3 \\
\hline 13 & & $X-1$ & $X-1$ & $X-1$ & & & 4 \\
\hline 14 & $X-1$ & & & & & $X-1$ & 2 \\
\hline 15 & $X-1$ & & & & & $X-1$ & 2 \\
\hline 16 & & $X-1$ & $X-1$ & $\mathrm{X}-1$ & & $X-1$ & 4 \\
\hline 17 & & $X-1$ & $X-1$ & $X-1$ & & $X-1$ & 4 \\
\hline 18 & & $X-1$ & & & & $X-1$ & 2 \\
\hline 19 & & $X-1$ & & & $X-1$ & $X-1$ & 3 \\
\hline 20 & $X-1$ & & & & & $X-1$ & 2 \\
\hline Use by Value & 8 & 14 & 6 & 5 & 4 & 9 & \\
\hline
\end{tabular}

Nursing nearing the end of the final semester of the year.

These students were from the September 2014 cohort and had been assessed using personal statements (during screening) and structured selection interviews. A number of the cohort $(\mathrm{N}=110)$, of which our volunteers were a sub-set $(\mathrm{N}=63)$, had volunteered to participate in a pilot of an MMI value based assessment process (based on the same nursing values used in the NM instrument). They were therefore well- positioned to provide feedback on the MMI and NM Values Based Recruitment (VBR) selection processes.

The NM study was held after the MMI pilot procedure that took place on the 23rd March 2015. Those respondents $(\mathrm{N}=110)$ who had taken part in the MMI study had been asked by School of Nursing staff if they were willing to participate and they were offered the inducement of a free lunch of sandwiches and coffee and participation in a draw 
for retail vouchers of $£ 100$ and $£ 200$ respectively. Sixty-three students agreed to participate.

\section{Procedure}

All the September 2014 cohort entering the School of Nursing were given Student Unique Identifier (SUI) numbers which were used to identity their personal statement scores, their initial interview scores, their MMI scores and their scores on the NM VBR instrument and, subsequently, their scores on end of year modules.

On 5th May 2015, 63 first year students completed the NM pilot instrument in a group setting (a computer laboratory).

The Ipseus software was downloaded and the NM instrument was completed by all 63 respondents each of whom sat at a desk at their own terminal well-spaced out in a computer room.

A presentation was delivered to brief all respondents on the procedure to be followed. Respondents were requested not to consult on responses.

Immediately after completion of the instrument each respondent completed a feedback questionnaire (Appendix C). A free text box was available.

\section{Results}

\section{Values based appraisal: data output}

The full set of data on the respondents' appraisals is set out, rank ordered, in Appendix A: Table 2. A subset of the data with mean scores is presented in Table 4 below as an illustration of the nature of output for assessment or screening purposes.

The outcome is an $S^{\mathrm{TOT}}$ score for each nursing value and a mean $S^{\text {TOT }}$ score for each respondent for the set of six values.

The results for individuals can be easily compared with scores for the cohort. Either as a simple rank ordering as in Appendix A: Table 2 or they can be presented in a more informative manner as individual or cohort scores (Figure 1).

\section{Statistical properties of the data}

Table 5 details a full range of responses and similar standard deviation of nursing values (SD).

$S^{\text {Tот }}$ scores on every major value have a distribution that approximates to normal. Because of constraints on space only the histogram of the mean of individual scores on all six major values is offered as evidence see Figure 2 (one outrider removed).

\section{Secondary correlation study}

A subsidiary study explored the correlation of the NM scores with measures of nursing competence: personal statements, selection interviews, end year module scores and an MMI selection process. Work underway on correlation seems to be suggesting that there is virtually no linear relationship between appraisal measures - see Table 6 which typifies what is being found. They do not appear to be measuring the same thing. The relationship between values and observed behavior is indeed a complex one to the extent of invalidating the whole VBR process some researchers would say. Other measures seem to be mired in the same bog of complexity in human behavior over time. I am not keen to highlight the fact that NM inhabits the same 'twilight zone'.

Feedback: the responses to the questionnaires (see the questionnaire at Appendix C) on the experience of completing the pilot instrument were collated and the data on responses to the questions analysed. See comment below and Table 7 below for a summary of the findings and comparison with the MMI feedback.

Text from the 'free text box' was reviewed and the findings summarised in Appendix A: Table 8.

Summary of feedback: the NM instrument was seen by respondents to:

- $\quad$ have face value and

- $\quad$ identify most important nursing values,

- $\quad$ be interesting,

\begin{tabular}{|l|r|}
\hline 0027 Characteristic nursing values & $\mathbf{S}^{\text {TOT }}$ score (\%) \\
\hline Person Centredness (PC) & 68.00 \\
\hline Accountability (ACC) & 66.39 \\
\hline Trust (T) & 61.79 \\
\hline Inte grity (I) & 59.91 \\
\hline Commitment Personal Development (CPD) & 55.54 \\
\hline Teamwork (TW) & 50.61 \\
\hline Mean & 60.37 \\
\hline Cohort: Characteristic nursing values & Mean S ${ }^{\text {ToT }}$ score (\%) \\
\hline Person Centredness (PC) & 71.00 \\
\hline Accountability (ACC) & 58.33 \\
\hline Trust (T) & 56.75 \\
\hline Integrity (I) & 52.59 \\
\hline Commitment Personal Development (CPD) & 56.38 \\
\hline Teamwork (TW) & 58.27 \\
\hline Mean & 58.89 \\
\hline
\end{tabular}

Figure 1. One respondent's scores compared with cohort scores.

Table 4. Section of $\mathrm{NM}^{\mathrm{m}}$ values based results table before rank ordering.

\begin{tabular}{|c|c|c|c|c|c|c|c|}
\hline Student & $\begin{array}{l}\text { Person Centredness } \\
\text { (PC) }\end{array}$ & Accountability (ACC) & Trust (T) & Integrity (I) & $\begin{array}{c}\text { Commitment Personal Development } \\
\text { (CPD) }\end{array}$ & Teamwork (TW) & Mean \\
\hline SUI0001 & 85.61 & 61.48 & 50.76 & 54.48 & 51.59 & 60.96 & 60.81 \\
\hline SUI0003 & 76.81 & 68.98 & 70.90 & 57.47 & 72.13 & 58.75 & 67.51 \\
\hline SUI0004 & 32.34 & 13.80 & 7.07 & -12.90 & 32.71 & 18.34 & 15.22 \\
\hline SUI0005 & 46.42 & 35.19 & 24.25 & 38.34 & 43.14 & 33.70 & 36.84 \\
\hline SUI0006 & 54.58 & 43.23 & 36.03 & 45.08 & 35.65 & 39.85 & 42.40 \\
\hline SUI0007 & 82.32 & 62.52 & 65.57 & 40.21 & 89.73 & 68.26 & 68.10 \\
\hline
\end{tabular}


Table 5. Simple descriptive statistics for the $\mathrm{S}^{\mathrm{TOT}}$ scores on the themed values.

\begin{tabular}{|l|c|c|c|c|}
\hline Nursing Value & Min & Max & Mean & SD \\
\hline Person Centredness (PC) & 32 & 98 & 73 & 14 \\
\hline Accountability (ACC) & 14 & 91 & 60 & 15 \\
\hline Trust (T) & 7 & 98 & 58 & 19 \\
\hline Integrity (I) & -13 & 93 & 54 & 19 \\
\hline $\begin{array}{l}\text { Commitment Personal Development } \\
\text { (CPD) }\end{array}$ & 9 & 94 & 58 & 19 \\
\hline Teamwork (TW) & 18 & 90 & 60 & 14 \\
\hline Mean & 15 & 93 & 60 & 15 \\
\hline
\end{tabular}

Table 6. Pearson Correlation between $\mathrm{S}^{\mathrm{TOT}}$ scores on NM themed values.

\begin{tabular}{|c|c|c|c|c|c|c|c|}
\hline \multicolumn{7}{|c|}{ Moderate to strong correlations: $\mathrm{p}$-Value $=0.000$ for all } \\
\hline PC & & 0.85 & 0.695 & 0.716 & 0.515 & 0.85 & 0.852 \\
\hline ACC & 0.85 & & 0.886 & 0.868 & 0.758 & 0.866 & 0.984 \\
\hline T & 0.695 & 0.886 & & 0.762 & 0.79 & 0.724 & 0.928 \\
\hline I & 0.716 & 0.868 & 0.762 & & 0.458 & 0.836 & 0.872 \\
\hline CPD & 0.515 & 0.758 & 0.79 & 0.458 & & 0.513 & 0.779 \\
\hline TW & 0.85 & 0.866 & 0.724 & 0.836 & 0.513 & & 0.887 \\
\hline Mean & 0.852 & 0.984 & 0.928 & 0.872 & 0.779 & 0.887 & \\
\hline
\end{tabular}

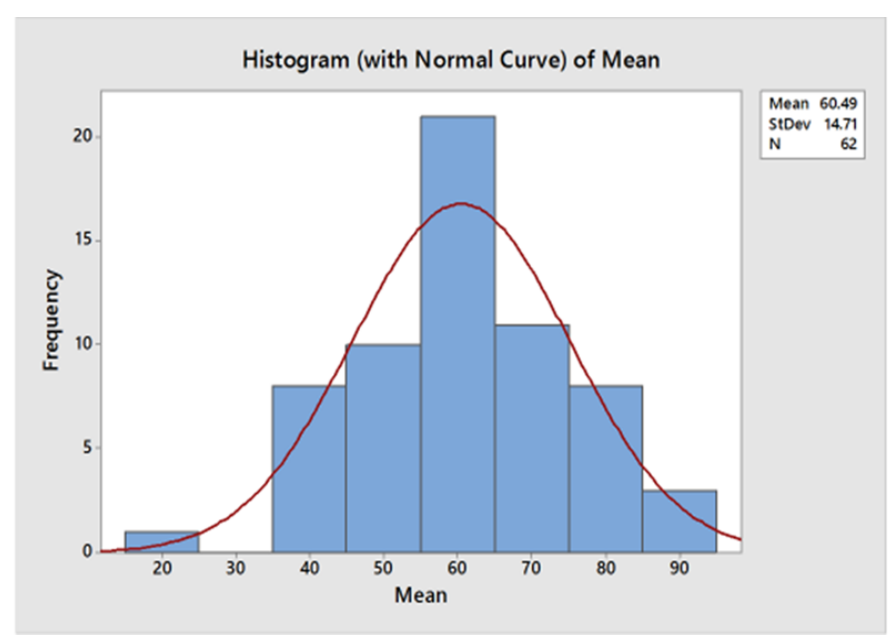

Figure 2. Distribution of mean scores on themed values.

Table 7. Weak negative or no linear relationship between NM values and MMI values.

\begin{tabular}{|c|c|c|c|c|c|c|c|c|}
\hline \multicolumn{9}{|c|}{ Pearson's r: $5 \%$ significance level } \\
\hline \multicolumn{9}{|c|}{ \% scores on MMI values } \\
\hline & & PC & ACC & $\mathbf{T}$ & I & CPD & TW & GLOBAL \\
\hline \multirow{14}{*}{$\begin{array}{l}\mathrm{S}^{\text {TOT }} \\
\text { scores } \\
\text { on NM } \\
\text { values }\end{array}$} & PC_1 & 0.17 & 0.25 & -0.09 & -0.08 & 0.11 & -0.12 & -0.11 \\
\hline & $\mathrm{p}$ value & 0.19 & 0.05 & 0.50 & 0.52 & 0.42 & 0.38 & 0.41 \\
\hline & ACC_1 & -0.11 & -0.24 & -0.09 & -0.13 & 0.15 & -0.12 & -0.04 \\
\hline & $\mathrm{p}$ value & 0.41 & 0.06 & 0.49 & 0.33 & 0.25 & 0.38 & 0.74 \\
\hline & T_1 & 0.01 & -0.19 & 0.02 & -0.02 & 0.12 & -0.11 & 0.07 \\
\hline & $\mathrm{p}$ value & 0.94 & 0.14 & 0.87 & 0.87 & 0.37 & 0.42 & 0.60 \\
\hline & I_1 & -0.08 & -0.28 & -0.14 & -0.11 & 0.11 & -0.04 & -0.05 \\
\hline & $\mathrm{p}$ value & 0.54 & 0.03 & 0.30 & 0.42 & 0.41 & 0.78 & 0.73 \\
\hline & CPD_1 & 0.08 & -0.07 & 0.06 & -0.05 & 0.17 & -0.07 & 0.09 \\
\hline & $\mathrm{p}$ value & 0.53 & 0.58 & 0.67 & 0.71 & 0.19 & 0.61 & 0.48 \\
\hline & TW_1 & -0.08 & -0.27 & -0.1 & -0.07 & 0.11 & -0.1 & -0.04 \\
\hline & $\mathrm{p}$ value & 0.56 & 0.04 & 0.46 & 0.57 & 0.41 & 0.44 & 0.74 \\
\hline & MEAN_1 & -0.05 & -0.24 & -0.06 & -0.09 & 0.15 & -0.1 & -0.01 \\
\hline & $\mathrm{p}$ value & 0.68 & 0.06 & 0.67 & 0.52 & 0.27 & 0.45 & 0.97 \\
\hline
\end{tabular}

Note: - indicate Centre Pearson r heading
Table 8. MMI and NM: feedback from students compared.

\begin{tabular}{|l|l|l|l|}
\hline & MMI & NM & \\
\hline Student comment (N = 110) & $\%$ & $\%$ & Student comment (N = 63) \\
\hline A positive experience & 86 & 98 & Easy to complete \\
\hline A fair assessment tool & 79 & 95 & Easy/mostly easy to understand \\
\hline Tested their suitability for profession & 74 & 94 & No key nursing values missing \\
\hline $\begin{array}{l}\text { Could show understanding better than } \\
\text { interview }\end{array}$ & 71 & 90 & Interesting to complete \\
\hline $\begin{array}{l}\text { Better way to select than current style } \\
\text { of interview }\end{array}$ & 58 & 90 & Not too challenging to complete \\
\hline Unsure about this & 31 & 84 & Responses easy intuitive \\
\hline & & 83 & Issues raised were important \\
\hline ASSESSORS COMMENT & & 81 & All questions asked made sense \\
\hline Wide range of attributes & 92 & 81 & Not hard work sometimes testing \\
\hline Appropriate way of Assessing & 81 & 10 & Had a little bit of difficulty here and there \\
\hline & & 8 & Felt they needed more time to complete \\
\hline & & & Free text \\
\hline & & & Different but easier than interviews/MMI \\
\hline & & & Better or worse was conflicted \\
\hline
\end{tabular}

- $\quad$ be easy to understand and complete and

- $\quad$ it was said to be a 'different experience'.

Some concern was expressed about

- $\quad$ the usefulness of a self-response instrument in an assessment of values compared with an assessment of responses to 'real situations' (contrived) as in the MMI stations.

Note: the two processes appear to measure different things and are complementary

- the MMI assessment being based on subjective observation and scoring on of a set of values,

- $\quad$ the Nurse Match VBR process being a self-report assessment using the same set of values.

\section{Discussion}

The primary aim of the work was to continue development of NM by piloting the instrument with a cohort of nurses, scoring the resulting value profiles and obtaining feedback on user experience.

The choice of NM values (nursing attributes) was found to be well aligned with recently researched attributes and six value themes.

Presentation and use of the instrument worked well and the scoring process for comparing respondent profiles on nursing values discriminated effectively between nursing students. Statistically it produced a normal distribution of scores overall, and for each major value, with means, range of scores and variance that were psychometrically acceptable.

The instrument was seen by respondents to have face and content validity identifying the important nursing values, was interesting, easy to understand and complete and was said to be a different experience. As a self-report measure it was considered to be complementary to other modes of assessment such as MMI.

Just asking people directly about themselves can offer revealing, fascinating and rich data. By their very nature these internal states and perceptions are not easily assessed by direct observation. However valid self-reports rely on self-awareness, personal honesty and good judgement so, particularly with young people, there may only be modest, if any, agreement between construal of self and appraisal by others. 
There is clearly a need for complementarity in appraisal of nurses and candidates for both developmental and recruitment purposes.

A secondary aim was to explore the relationship between cohort scores on the pilot instrument and a number of other measures of nursing competence; personal statements, selection interviews and an MMI selection process.

Within the appraisal processes such as NM and MMI used in this project there is clear evidence of positive linear relationships between measures of value themes. This seems reassuring about validity.

However, while this secondary aim is a work in progress, initial indications are of low or no linear relationship, positive or negative, between the various measures of nursing competence. It seems that each mode of assessment has been of practical use in recruitment and selection but each says something different about the characteristics of the nurse or candidate and their potential as nurses.

The most common approach used at present for an initial screening of values is a Personal Statement written by the candidate and assessed by expert markers. This is a questionable procedure with regards to validity, reliability and feasibility. On the basis of the work completed on NM and reported in this paper and Ellis et al. [1] it is suggested that Nurse Math is a more valid, reliable and cost effective method for initial screening of applicants than the Personal Statement.

The lack of a positive linear relationship between measures may be because there is no empirical evidence to suggest that future performance in the role of nurse can be predicted with any certainty and so serendipity rules.
However, it seems that someone with an appropriate set of nursing values today will probably perform more effectively later in life than someone with a poor set of nursing values today. On the evidence of this piece of work, NM can be a most effective, efficient and systematic way to get at and assess those values.

\section{References}

1. Ellis R, Griffiths L, Hogard E (2015) Constructing the Nurse Match Instrument to Measure Professional Identity and Values in Nursing. Journal of Nursing and Care 4: 245.

2. Northern Ireland Practice and Education Council (NIPEC) for Nursing and Midwifery (2014) Gateway to Nursing: Report of Phase 2: Attributes which are Valued to Support Career in Nursing. NIPEC: Belfast.

3. Great Britain. Department of Health (2014) Delivering high quality, effective, compassionate care: developing the right people with the right skills and the right values. A mandate from the government to Health Education England: April 2015 to 2016. Social Care Online.

4. Department of Health (2015) The NHS constitution for England. London.

5. Health Education England (HEE) (2014) Values Based Recruitment Framework. HEE: London.

6. Francis R (2013) Report of the Mid Staffordshire NHS Foundation Trust Public Inquiry. The Stationery Office, London.

7. Nursing and Midwifery Council (NMC) (2015) The Code. Professional standards of practice and behaviour for nurses and midwives. NMC: London.

8. Department of Health (2010) Essence of Care 2010 (Best Practice Guidance: Gateway 14641) The Stationery Office: UK

9. Passmore G, Ellis R, Hogard E (2014) Measuring Identity: A Review of Published papers SHEU Occasional paper 12 Buckinghamshire New University.

10. Weinreich P, Saunderson W (2012) Analysing Identity: Cross-Cultural, Societal and Clinical Contexts. RKP, London.

Copyright: (C2016 Hogard E. This is an open-access article distributed under the terms of the Creative Commons Attribution License, which permits unrestricted use, distribution, and reproduction in any medium, provided the original author and source are credited. 